\title{
COEXISTENCIA DE ASPERGILOSIS E HIDATIDOSIS PULMONAR. REPORTE DE UN CASO
}

\author{
Karen Yanet Castillo-Minaya ${ }^{1, a}$, Ana Cecilia Cherre-Fiestas ${ }^{1, a}$, José Rene Somocurcio-Peralta ${ }^{2, b}$
}

\begin{abstract}
RESUMEN
La hidatidosis es una zoonosis causada por el parásito Echinococcus granulosus. Perú es una zona endémica para esta infección, presentamos el caso de una mujer de 33 años, procedente de la región de lca; que durante la gestación se le diagnosticó hidatidosis hepática y pulmonar y posterior al término de la gestación fue referida con tubo de drenaje torácico, disnea y dolor torácico en hemitórax derecho, de curso progresivo e imagen tomográfica de tórax que mostró quiste pulmonar basal posterior derecho. Los exámenes serológicos fueron positivos, la paciente fue sometida a resección quirúrgica con resultado histopatológico de quiste hidatídico pulmonar complicado no viable y estructura micótica consistente con aspergiloma. La coexistencia de estas dos entidades es rara y pueden ser amenazas potenciales para los pacientes. El diagnóstico y tratamiento tempranos son vitales para prevenir posibles complicaciones como la hemoptisis masiva o incluso enfermedad invasiva.
\end{abstract}

Palabras clave: Equinococcus granulosus; Equinococosis; Zoonosis; Aspergillus (fuente: DeCS BIREME).

\section{COEXISTENCE OF PULMONARY ASPERGILLOSIS AND HYDATIDOSIS. CASE REPORT}

\begin{abstract}
Hydatidosis is a zoonosis caused by the parasite Echinococcus granulosus and Peru is an endemic zone for this infection. We present the case of a 33-year old woman from the region of Ica who, during gestation, was diagnosed with liver and pulmonary hydatidosis. After gestation was terminated, she was referred with thoracic drainage tube, dyspnea, and thoracic pain in right hemithorax, with progressive course and tomographic image of thorax showing right posterior basal pulmonary cyst. The serological analysis was positive, the patient underwent surgical resection with histopathologic result as complex non-viable pulmonary hydatidic cyst and mycotic structure consistent with aspergilloma. The coexistence of these two diseases is rare and can mean potential threats for patients. The early diagnosis and treatment are vital to prevent possible complications such as massive hemoptysis or even invasive disease.
\end{abstract}

Keywords. Equinococcus granulosus; Echinococcosis; Zoonoses; Aspergillus (source: MeSH NLM).

\section{INTRODUCCIÓN}

La hidatidosis es una enfermedad zoonótica causada por el parásito Echinococcus granulosus. Afecta principalmente a los perros, hospedador definitivo donde se desarrolla la fase adulta, así como a bovinos, ovinos y porcinos (hospedadores intermediarios en donde se desarrolla la fase larvaria o quiste hidatídico). La infección en humanos ocurre tras la ingestión accidental de los huevos del parásito. En el intestino delgado del humano se produce la disolución de la cubierta de los huevos del parásito, y se liberan embriones que atraviesan la mucosa intestinal y pasan a la circulación venosa para llegar a los diferentes órganos. La mayor frecuencia de localización es la hepática $(67-89 \%)$, seguida de la pulmonar $(10-15 \%)^{(1,2)}$.
La equinococosis quística en Sudamérica tiene gran incidencia en países como Argentina, Brasil, Uruguay, Chile y Perú (3), en este último se reporta 64 casos por 100000 habitantes ${ }^{(4)}$. También se han reportado casos durante la gestación, pero su incidencia es menor y se calcula de 1 en 20000 a $30000^{(5)}$. Entre las complicaciones de la hidatidosis quística destacan la ruptura del quiste, normalmente hacia las cavidades peritoneal o pleural; lo que puede dar lugar tanto a la diseminación de la infección a otros órganos como al desarrollo de reacciones anafilácticas. También es posible la aparición de infecciones micóticas, bacterianas e incluso abscesos en el seno o en el entorno de los quistes ${ }^{(6)}$.

Existen pocos reportes en la literatura médica sobre la coexistencia de quiste hidatídico con aspergilosis. Aspergillus

\footnotetext{
Universidad Peruana Cayetano Heredia. Lima, Perú.

Hospital Edgardo Rebagliati Martins, EsSalud. Lima, Perú.

a Médico residente de Patología y Laboratorio Clínico; ${ }^{b}$ Médico Anatomopatólogo.

Recibido: 31/01/2018 Aprobado: 24/10/2018 En línea: 21/12/2018
} 
es un hongo saprofítico, se han identificado alrededor de 200 especies, sin embargo, muy pocas especies causan enfermedad en humanos, el Aspergillus fumigatus es la especie patógena más común. La presencia de lesiones cavitarias en el pulmón es un factor predisponente para la infección por Aspergillus, que tiende a crecer en áreas con tejido desvitalizado y sus factores predisponentes son la inmunodeficiencia y alteraciones en el sistema pulmonar ${ }^{(7,8)}$.

La infección consiste en una masa de hifas fúngicas, células inflamatorias, fibrina, moco y restos de tejido y puede colonizar el pulmón debido a enfermedades subyacentes tales como tuberculosis, sarcoidosis, bronquiectasias, bullas, cáncer de pulmón cavitario, neoplasias, espondilitis anquilosante, quistes bronquiales, infarto pulmonar, etc. (7). El hígado y los pulmones son los sitios más comunes de esta infección. La mayoría de los casos reportados fueron hombres $(70 \%)$, y eran inmunocompetentes, en pacientes inmunocomprometidos se presenta con una hemoptisis potencialmente mortal ${ }^{(2)}$.

Reportamos el siguiente caso por la presentación inusual de estas entidades y la importancia clínica para su manejo.

\section{REPORTE DEL CASO}

Mujer de 33 años, procedente de la ciudad de Ica, en la región de Ica, Perú, con grado de instrucción superior, trabajó en actividades de agronomía y biología. Niega otros antecedentes patológicos y epidemiológicos.

La historia clínica refiere que en su cuarto mes de gestación presenta un cuadro de hiperemesis gravídica y en controles de imagen se detectan lesiones quísticas en segmento hepático VII de 53x46x66 mm e incidentalmente quiste supradiafragmático derecho de $68 \times 71 \mathrm{~mm}$, dos informes particulares de Western blot fueron positivos para Echinococcus granulosus; asintomática en controles regulares, fue candidata a cirugía electiva posterior a término de gestación.

En la historia no se menciona si recibió tratamiento farmacológico previo a la intervención quirúrgica pues fue referida por consultorio externo, dos meses posteriores al término de la gestación para evaluación y manejo quirúrgico.

En un informe tomográfico de tórax se evidenció un quiste pulmonar basal posterior derecho de ubicación periférica de paredes delgadas con contenido líquido homogéneo de aproximadamente $70 \times 72 \times 60 \mathrm{~mm}$ sin componente inflamatorio contiguo de aspecto simple.

Diez días antes del ingreso presentó malestar general, fiebre, se agregó disnea y dolor torácico en hemitórax derecho, le colocaron tubo de drenaje torácico y fue referida de emergencia a la ciudad de Lima, con un diagnóstico de quiste hidatídico pulmonar complicado en hemitórax derecho.

En el examen físico se evidenció un tórax simétrico, murmullo vesicular disminuido y tubo de drenaje torácico con una secreción turbia. En una tomografía abdominal se evidenció la presencia lesión hipodensa de 47x29 mm con una calcificación en su interior a nivel del segmento VII hepático (Figura 1).

Es intervenida quirúrgicamente y se evidencia lóbulo superior y medio derecho atrapado por tejido pleural grueso, quiste hidatídico gigante roto a cavidad pleural y un quiste periférico que dependía de lóbulo inferior derecho. Membranas y secreciones purulentas libres en cavidad de aproximadamente $300 \mathrm{cc}$.

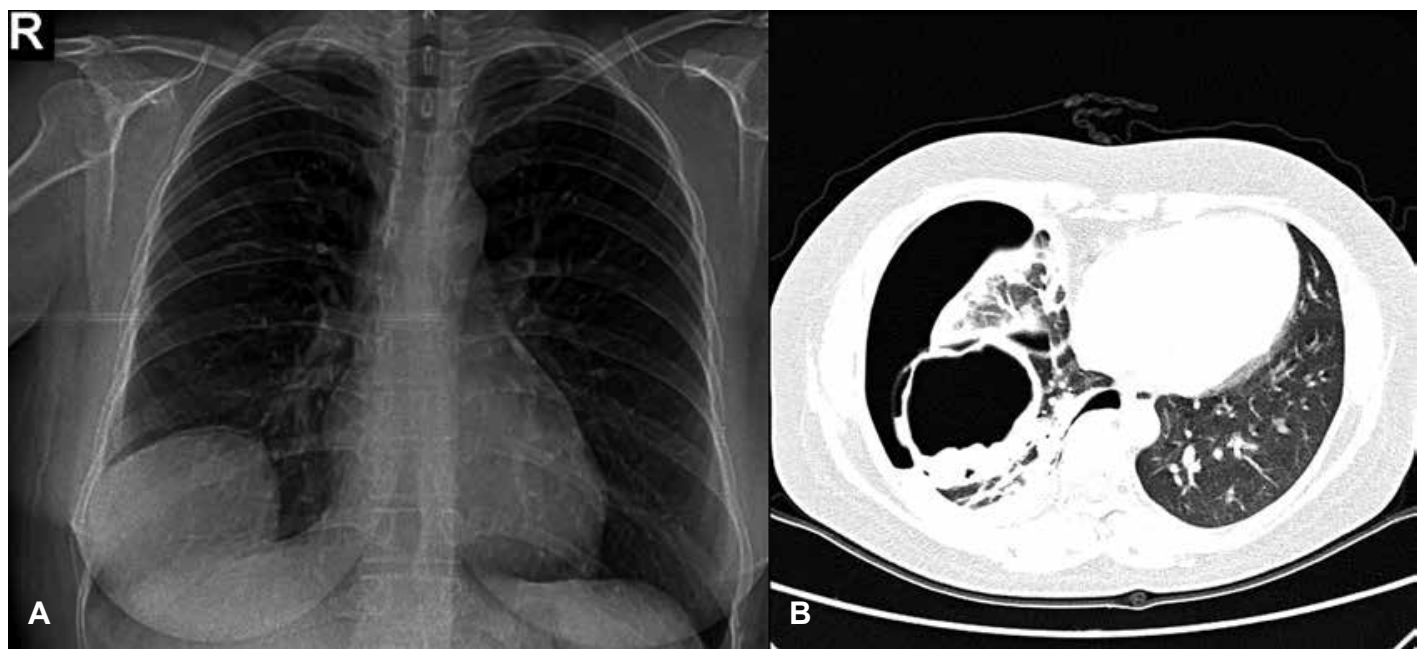

Figura 1. A. Radiografía de tórax: Imagen de aspecto cavitario de pared gruesa con borde externo irregular proyectada en la base del pulmón derecho que obtura el seno costodiafragmático respectivo. B. Tomografía de Tórax: Quiste pulmonar basal posterior derecho de ubicación periférica de paredes delgadas con contenido líquido homogéneo 
En el servicio de anatomía patológica se recibieron múltiples fragmentos de tejido pardo amarillento, de aspecto hemorrágico que en conjunto pesaron $200 \mathrm{~g}$. El mayor midió $7 \times 6 \times 1 \mathrm{~cm}$ y el menor midió $0,3 \times 0,2 \times 0,2 \mathrm{~cm}$. La microscopía fue informada como quiste hidatídico pulmonar complicado no viable y estructura micótica consistente con aspergiloma (Figura 2).

Se realizaron tinciones histoquímicas como tricrómica donde se observaron los conidios (Figura 3) y coloración de plata metenamina donde se evidenció que el Aspergillus colonizó las membranas hidatídicas (Figura 4).

Posterior a la intervención quirúrgica se le indicó tratamiento farmacológico con albendazol $200 \mathrm{mg}$, dos tabletas cada 12 horas y una tomografía control sin contraste; la que se realizó un mes y medio después; por estudios comparativos con exámenes previos se evidenció resolución de la lesión quística pulmonar derecha, así como evacuación del hidroneumotórax ipsilateral identificándose atelectasias retráctiles secuelares de menor magnitud comparado a estudios previos. No se identificó nueva lesión quística intrapulmonar. En cortes representativos del abdomen superior persiste lesión quística heterogénea en los segmentos VII-VIII en relación al antecedente referido y con leve disminución de sus dimensiones con un diámetro mayor de $34 \mathrm{~mm}$, siendo en el control previo de $55 \mathrm{~mm}$.

Un año posterior a la intervención quirúrgica se encuentra asintomática, continúa en tratamiento con albendazol $200 \mathrm{mg}$, 2 tabletas cada 12 horas y controles de transaminasas normales: aspartato aminotransferasa (AST) $(16 \mathrm{U} / \mathrm{L})$ y alanina transaminasa (ALT) $(15,6 \mathrm{U} / \mathrm{L})$.

La última tomografía de tórax informa tractos secuelares en el pulmón derecho; a predominio derecho leve elevación del diafragma derecho. No efusión pleural, no adenomegalias

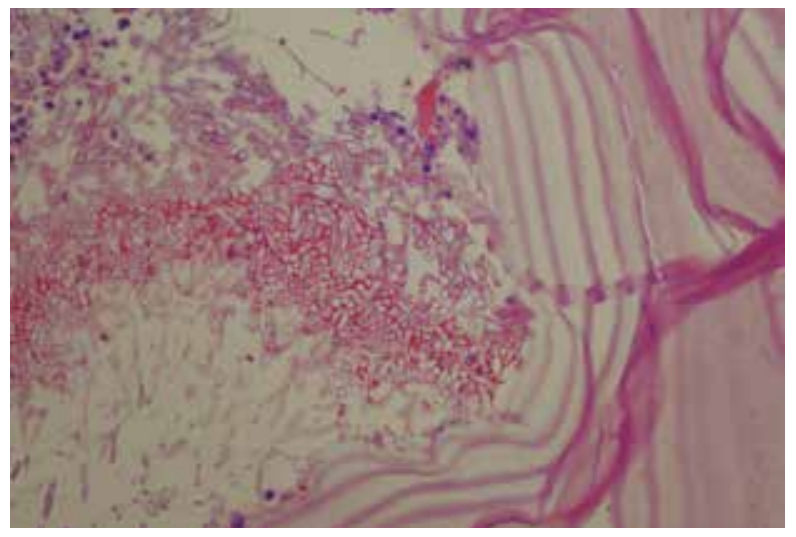

Figura 2. Pared de la membrana acelular laminada de un quiste hidatídico colonizado por Aspergillus spp; hifas fúngicas uniformes, tubulares, regularmente septadas, ramificándose dicotómicamente, además de moderada cantidad de células inflamatorias, tinción $\mathrm{H}-\mathrm{E}$, aumento $20 \mathrm{X}$ significativas, mediastinales ni axilares. No se delimita la presencia de lesiones quísticas. Incidentalmente se observa lesión quística en el nivel hepático.

\section{DISCUSIÓN}

La coexistencia de aspergilosis y equinococosis es extremadamente rara. En un estudio de 100 casos con diagnóstico de quiste hidatídico, solamente en dos pacientes presentaron esta coexistencia ${ }^{(8)}$. En Perú es el primer caso documentado; se han hecho reportes de la coexistencia de aspergilosis en casos de tuberculosis pulmonar ${ }^{(7,9-11)}$; como el reportado por Arce en 14 pacientes con tuberculosis en la región de Ica ${ }^{(9)}$. En el reporte se tuvieron dos resultados positivos de Western blot dado que la paciente tenía hidatidosis hepática y pulmonar.

En este reporte el diagnóstico inicial se hace como un hallazgo incidental durante una ecografía de rutina al tratarse de una mujer gestante. Sin embargo, se debe tener en cuenta algunas características epidemiológicas como el lugar de procedencia y las actividades en la que laboraba la paciente como fueron agronomía y biología. La presentación clínica de los quistes hidatídicos pulmonares depende si el quiste está intacto o roto. Un quiste complicado se define como uno que se ha roto en un bronquio, cavidad pleural o árbol biliar. Los quistes intactos son hallazgos incidentales o se acompañan de algunos síntomas como tos, disnea o dolor en el pecho.

La historia clínica no refiere tratamiento médico ni el mecanismo del término de la gestación; pues fue referida por una complicación del quiste hidatídico. La gestación podría favorecer la ruptura de quistes hidatídicos por el crecimiento del útero grávido que incrementa la presión intraabdominal e intratorácica. El quiste hidatídico hepático crece a un promedio de $1 \mathrm{~cm}$ al año (12); además del riesgo de anafilaxia, parto pre término entre otros ${ }^{(9)}$. No existe un consenso de manejo específico durante la gestación y existen pocos reportes; pero refieren que el tratamiento de la hidatidosis pulmonar es quirúrgico y en gestantes mayores de 25 semanas se recomienda la intervención quirúrgica después de una cesárea programada a las 34 semanas ${ }^{(13)}$. El empleo de mebendazol y albendazol es útil en el tratamiento primario de la hidatidosis y en la prevención de recurrencias posoperatorias en no embarazadas. Sin embargo, su uso en gestantes no es recomendado pues tienen riesgo teratogénico y embriotóxico ${ }^{(14)}$.

En el reporte se indica que el tratamiento con albendazol fue indicado posterior al parto e intervención quirúrgica, no hubo eventos significativos en cuanto mortalidad en la paciente. Siendo así que la cirugía ofrece la única opción curativa para estas pacientes, y consiste en la evacuación del contenido seguido de esterilización y exéresis de las membranas, o en la resección anatómica o no anatómica 


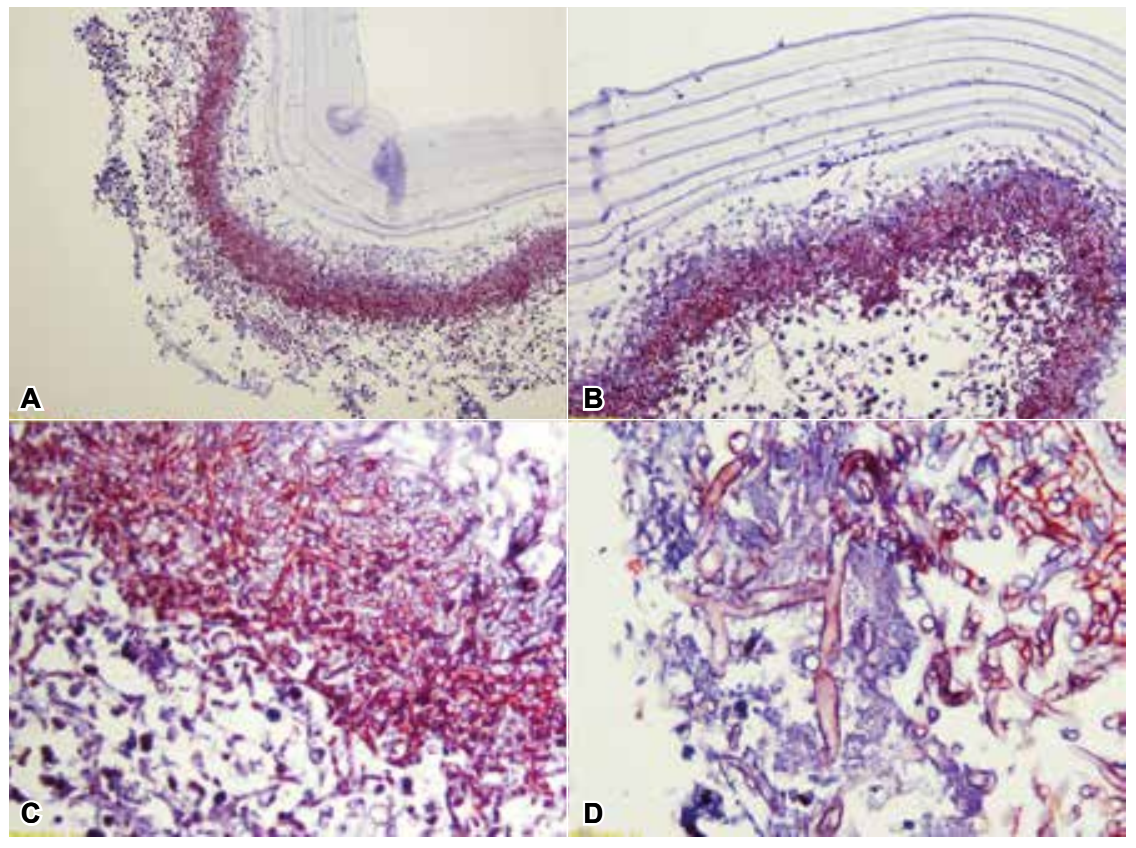

Figura 3. Tinción Tricrómica donde se observan células inflamatorias mixtas. La membrana acelular laminada del quiste hidatídico y la morfología hifal del Aspergillus. A. Aumento 5X, B. Aumento 10X, C Aumento 20X y D Aumento 40X

que incluya el quiste hidatídico ${ }^{(15)}$. La disnea, dolor torácico, fiebre fueron las quejas principales similar a lo que reportó Pan et al. donde el paciente se sometió a cirugía para evitar el riesgo de anafilaxia y diseminación ${ }^{(15)}$.
Los quistes hidatídicos pueden romperse espontáneamente o como resultado de un traumatismo o una infección secundaria. Pueden infectarse con bacterias, saprofitos o con hongos, como se ha visto en el presente caso. Los

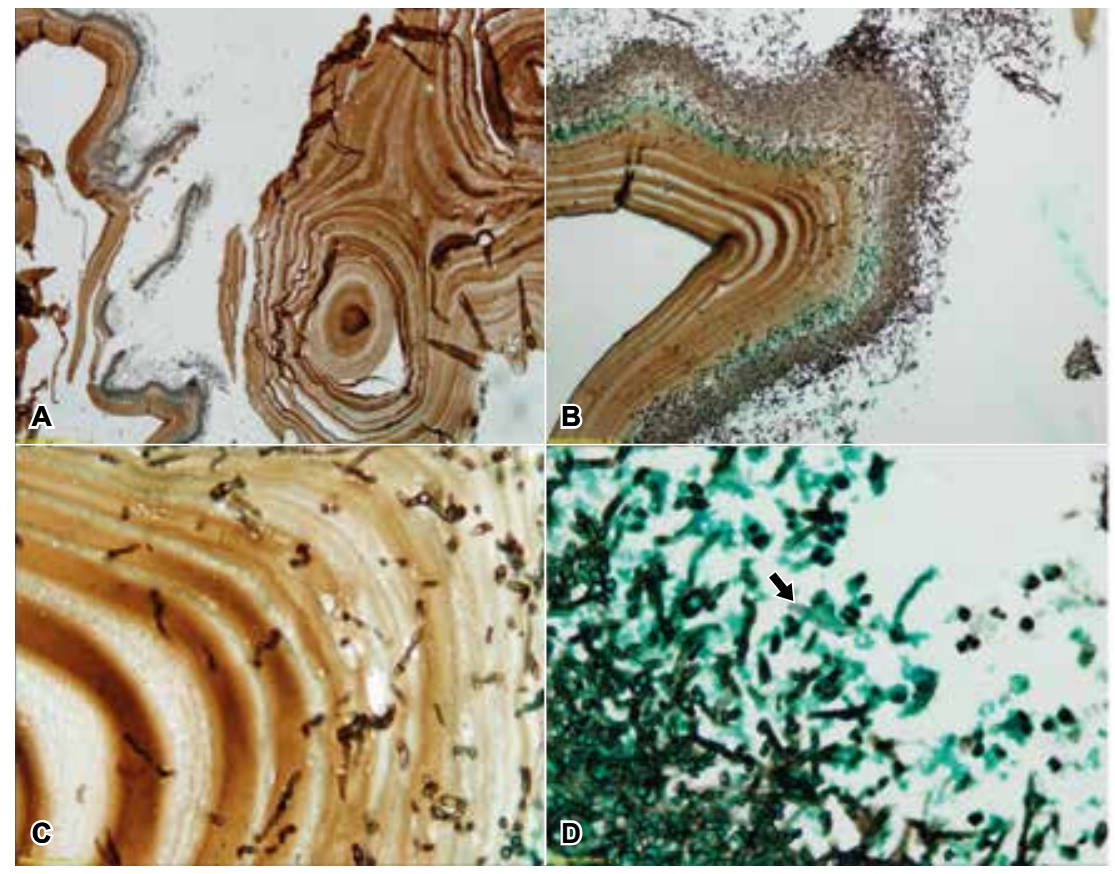

Figura 4. Coloración de plata metenamina: Se observan hifas de Aspergillus que invaden el quiste hidatídico pulmonar (a. Aumento $5 \mathrm{X}$ y b. Aumento 10X). Hifas septadas con ramificación en ángulo de 450 (b y c Aumento 40X) y en d. Aumento 40X: un gancho característico de la especie E. granulosus (flecha). 
hongos pueden invadir la capa externa de la membrana hidatídica y pueden crecer en la cavidad (11). El quiste equinocócico que probablemente se comunicó con el bronquio podría haber sido la causa de la infección con Aspergillus. Sin embargo, en casos de quistes rotos, especialmente en pacientes inmunocomprometidos, el paciente puede adquirir la infección, incluso avanzar a la forma invasiva. Esta coexistencia única debido a su rareza hace que tenga una importancia clínica para su manejo ${ }^{(15)}$.

El diagnóstico y tratamiento tempranos son vitales para prevenir complicaciones derivadas de la infección por estos dos patógenos, como la hemoptisis masiva o incluso enfermedad invasiva (11). Sin embargo, en casos de quistes rotos, especialmente en pacientes inmunocomprometidos, el paciente puede estar en riesgo de una infección por Aspergillus, pudiendo incluso avanzar a la forma invasiva ${ }^{(15)}$. Para un adecuado tratamiento, el médico debe tener en cuenta la individualidad de cada paciente, a fin de poder identificar y evaluar aquellas situaciones particulares.

En la hidatidosis pulmonar está indicado exclusivamente el tratamiento quirúrgico sean estos sintomáticos o no; a diferencia de la hidatidosis hepática donde existen otras opciones de tratamiento ${ }^{(16)}$. En la Aspergilosis el tratamiento definitivo es la resección quirúrgica que no sólo provee control sintomático, sino que confiere ventajas en supervivencia y se ha usado incluso en casos asintomáticos para prevenir el desarrollo de sangrado masivo ${ }^{(17)}$.

En conclusión, la coexistencia de estas dos entidades pueden ser amenazas potenciales para los pacientes, debido al diagnóstico difícil por la presentación infrecuente en una gestante con curso asintomático y el manejo posterior al término de la gestación necesita de una alta sospecha para predecirsuperposición demicosis. Los informes queaparecen en la literatura son escasos y existe poca información sobre el manejo óptimo de estas pacientes debido a los diferentes riesgos en relación a las complicaciones del quiste y la complejidad de los procedimientos quirúrgicos. El manejo debe ser multidisciplinario e involucra varias especialidades médicas.

Contribuciones de autoría: KYCM, ACCHF y JRSP han participado en la concepción del artículo, recolección de datos, en la redacción y aprobación de la versión final.

Fuentes de financiamiento: autofinanciado.

Conflictos de interés: Los autores declaran no tener conflictos de interés.

\section{REFERENCIAS BIBLIOGRÁFICAS}

1. Larrieu E. Prevención y control de la hidatidosis en el nivel local: iniciativa sudamericana para el control y vigilancia de la equinococosis quística / hidatidosis. Organización Panamericana de la Salud - OPS/OMS. Río de Janeiro: PANAFTOSA - OPS/OMS; 2017.

2. Aala F, Badali H, Hashemi Fesharaki $S$, Boroumand M, Sotoudeh Anvari M, Davari H, Agha Kuchak Afshari S, Khodavaisy S. Coexistence of aspergilloma and pulmonary hydatid cyst in an immunocompetent individual. J Mycol Med. 2017;27(3):396-399. doi: 10.1016/j.mycmed.2017.04.006.

3. Miranda E, Velarde F, Somocurcio J, Ayala E. Evaluación de dos pruebas de inmunoblot con antígeno Hidatídico de caprino y ovino para el diagnóstico de Equinococosis humana. Rev Peru Med Exp Salud Publica. 2010; 27(2): 209-14.

4. Salgado S, Suarez-Ognio L, Cabrera R. Características clínicas y epidemiológicas de la echinococosis quística registrada en un área endémica en los andes centrales del Perú (1991-2002). Neotrop Helminthol. 2007; 1(2):69-83.

5. López-Huamanrayme Eddy, Contreras-Sotomayor Shamir J, Suca-Saavedra
Ronald D, Cairo-Calderón Luis R. Hidatidosis pulmonar complicada en una mujer gestante: Reporte de caso y revisión de la literatura. Rev Med Hered. 2016; 27(1):172-176.

6. Agarwal S, Bohara S, Thakran A, Arora P, Singh R, Agarwal P N. Pulmonary hydatid disease with coexistent aspergillosis: An incidental finding. Indian J Med Microbiol. 2013;31(1):85-6. doi: 10.4103/0255-0857.108740.

7. Aliyali M, Badali H, Shokohi T, Moazeni M, Nosrati A, Godazandeh G, Nabili M. Coinfection of Pulmonary Hydatid Cyst and Aspergilloma: Case Report and Systematic Review. Mycopathologia. 2016;181(3-4):255-65. doi: 10.1007/s11046-015-9974-2.

8. Kocer NE, Kibar Y, Guldur ME, Deniz H, Bakir K. A retrospective study on the coexistence of hydatid cyst and aspergillosis. Int J Infect Dis. 2008;12(3):248-51.

9. Arce A, Guillermo J, Torres J, Casquero J. Aspergiloma pulmonar en el Hospital de Apoyo Departamental de Ica - Perú. 2000 - 2001. Rev Perú Med Exp Salud Publica. 2002;19(4):197-201.

10. Carrillo-Ñañez L, Canelo-Aybar C, Cuadra-Urteaga J, Zegarra-Del Alama- no Cinthya. Fiebre prolongada como manifestación de Aspergiloma pulmonar en un paciente con antecedente de tuberculosis. Rev Perú Med Exp Salud Publica. 2008;25(1):153-156.

11. Esquivel-Ramírez C, Duarte-Dávila A, González-Moncada C, SalablancaGaleano K. Aspergiloma en paciente con tuberculosis pulmonar activa. RCSEM. 2017;1(1): 57-62.

12. Espinosa J, Jover R, Ramírez R, Muci T, Lluís F. Quiste hidatídico hepático durante el embarazo. Cir esp. 2011; 90(1):54-67. doi:10.1016/j.ciresp.2010.07.019

13. Al-Ani A, Elzouki AN, Mazhar R. An imported case of echinococcosis in a pregnant lady with unusual presentation. Case Rep Infect Dis. 2013;4(3):77-84. https://doi. org/10.1155/2013/753848.

14. Erçetin C, Özden I, Iyibozkurt C, Güven K, Serin K, Bilge O, et al. Hepatic hydatid disease requiring urgent treatment during pregnancy. Ulus Travma Acil Cerrahi Derg 2013 Mar;19(2):119-22. doi: 10.5505/ tjtes.2013.21548.

15. Pan JB, Hou YH, Yin PZ. A case report of hydatid cysts containing Aspergillus. J Tho- 
rac Dis 2013;5(2): E25-E27. http://doi. org/10.3978/j.issn.2072-1439.2012.07.04

16. Moral M, Laplume H, Camera L, et al. Enfermedades infecciosas. Hidatidosis, Guía para el equipo de salud. Buenos Aires: Dirección de Epidemiologia/ Ministerio de Salud de la Nación Ar- gentina; 2012. Disponible en: http:// www.msal. gov.ar/images/stories/epidemiologia/pdf/guiamedica-hidatidosis.pdf

17. Stevens DA, Kan VL, Judson MA, Morrison VA, Dummer S, Denning DW, et al. Practices guidelines for dis- eases caused by Aspergillus. Clinic Infect Dis. 2000; 30(4): 696-709.

Correspondencia: Karen Yanet Castillo Minaya Dirección: Jr. Brigadier Pumacahua 1684 dpto. 403. Jesús Maria, Lima, Perú.

Correo electrónico:karen_yacami@yahoo.com

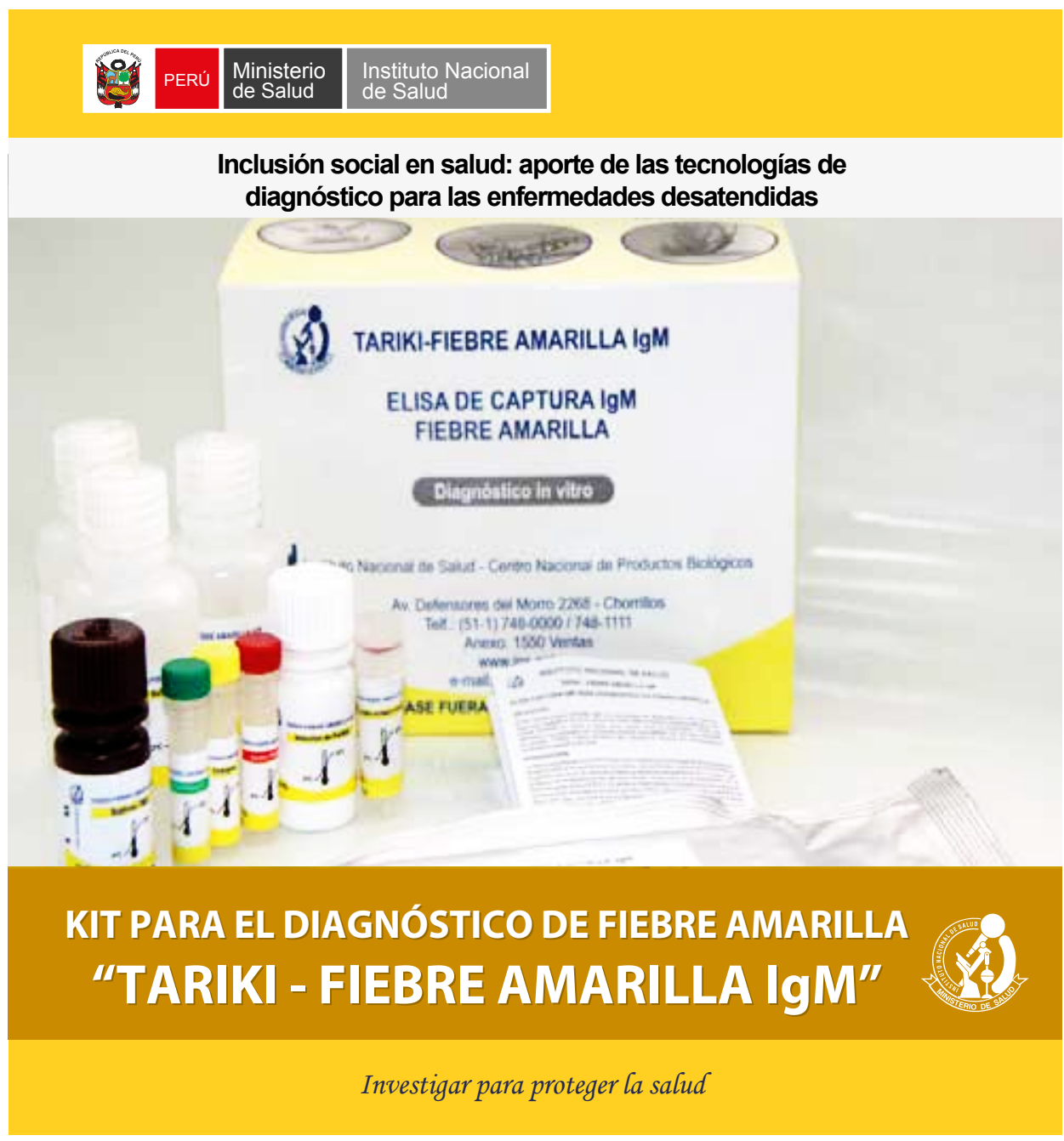

